\title{
Germanica
}

allemande : fascination et suspicion

\section{Le Docteur Faustus selon Giacomo Manzoni, du commentaire à l'œuvre scénique}

Laurent Feneyrou

\section{OpenEdition}

\section{Journals}

Édition électronique

URL : http://journals.openedition.org/germanica/1524

DOI : 10.4000/germanica.1524

ISSN : 2107-0784

Éditeur

Université de Lille

Édition imprimée

Date de publication : 30 juin 2005

Pagination : 115-124

ISBN : 9782913857155

ISSN : 0984-2632

Référence électronique

Laurent Feneyrou, «Le Docteur Faustus selon Giacomo Manzoni, du commentaire à l'œuvre

scénique », Germanica [En ligne], 36 | 2005, mis en ligne le 12 juillet 2012, consulté le 06 octobre 2020.

URL : http://journals.openedition.org/germanica/1524; DOI : https://doi.org/10.4000/germanica.1524

Ce document a été généré automatiquement le 6 octobre 2020.

(C) Tous droits réservés 


\title{
Le Docteur Faustus selon Giacomo Manzoni, du commentaire à l'œuvre scénique
}

\author{
Laurent Feneyrou
}

Dans la seconde moitié du xxesiècle, le mythe de Faust réinvestit la création musicale : Le Docteur Faustus de Thomas Mann en est la principale raison et, à travers lui, le dodécaphonisme schoenbergien. Composer un Faust signifie en effet affronter le spectre d'Arnold Schoenberg, l'un des modèles, mais non le seul, d'Adrian Leverkühn Dürer, et surtout Nietzsche s'introduisent, aisément identifiables, dans les lacis de l'œuvre. S'affirme aussi la volonté de retourner à un Faust antérieur à celui de Lessing, étranger à celui de Goethe si prisé par les musiciens du xixesiècle, et conscient de sa transformation mythique : ni l'enfer de Marlowe, ni l'éther goethéen. L'œuvre critique et musicale de Giacomo Manzoni, lecteur attentif de Thomas Mann, suscite un commentaire en des temps a priori incompatibles et supposant un système d'ouverture et d'occlusion qui tantôt les isole, tantôt les rend perméables : il y a le temps de Docteur Faustus, sous-titré « scene dal romanzo di Thomas Mann "(1988), œuvre en onze tableaux, un interlude et un épilogue issus d'une longue proximité avec la figure d'Adrian Leverkühn, celui de la préface que l'on vient de lire (1980) et celui de la lecture du roman, dès la publication de la traduction italienne d'Ervino Pocar en $1949^{1}$; il y a le temps de Sérénus Zeitblom, le narrateur qui entreprend son récit le 23 mai 1943, le jour même où Thomas Mann se consacre à la rédaction de son livre, et celui de Leverkühn découvrant le dodécaphonisme dans les années dix, avant la fameuse Valse de Schoenberg. Après le thème de la morale individuelle dans La sentenza (1960), après la désolation d'un monde atomisé (Atomtod, 1964), après l'idéal robespierriste de la Révolution française (Per Massimiliano Robespierre, 1974), Docteur Faustus, créé à la Scala de Milan le 16 mai 1989, dans une mise en scène de Robert Wilson, représente le rapport entre l'artiste et son œuvre, selon l'interprétation de Mann, cette "situation de l'art " dont le romancier faisait état dans une lettre à Theodor W. Adorno ${ }^{2}$, mais aussi le 
thème de l'individu, du sujet porteur d'instances transcendant la sphère restreinte du singulier.

Comment écrire un opéra sur Le Docteur Faustus, "roman de la musique »? Manzoni récusa toute solution de type cinématographique agréant la multiplication des personnages et des épisodes. Il s'en tint strictement au texte original, préleva de courts fragments, en style direct, susceptibles d'être confiés à des personnages d'opéra, et conserva initialement dans ses propres didascalies les mots de Thomas Mann - avant de privilégier des indications plus abstraites : «Intérieur », « Un lieu », « Un autre lieu »... Et selon Manzoni, dans le roman, les moments essentiels à la compréhension sont caractérisés par le discours direct, la conversation ou le soliloque. Divers renoncements à certains personnages et à certains chapitres, notamment dans les développements initiaux, éloignèrent Leverkühn de la reconstitution de son milieu social : le musicien reste seul face à lui-même, à la création, en proie à Lui, à ce Diable aux trois voix - une basse, comme le Pédiatre, un ténor léger, comme l'imprésario Saül Fitelberg, et une soprano, comme Esmeralda. L'intention de Manzoni était de laisser se dérouler l'action dans une dimension intemporelle et sans lieu. Georg Lukács a montré dans Brève histoire de la littérature allemande comment Thomas Mann opéra, en particulier dans La Montagne magique, une dissolution critique du temps et de l'espace social, dissolution caractéristique de la littérature allemande de l'Empire. "L'action est consciemment placée dans un milieu artificiellement isolé. Les hommes sont sans doute déterminés par leur psychologie sociale, mais se trouvent en dehors de leurs cadres sociaux habituels. Ainsi est créé un symbole ironique de la description sociale dans l'impérialisme allemand ${ }^{3}$." Partant, dans l'écriture même de son livret, Manzoni a mis en abîme ce procédé central de la littérature impériale, ce retrait, sinon ce déni du réel.

Leverkühn s'isole douloureusement de son peuple, où le sujet authentique n'existe que dans une collectivité fondée sur la fraternité, modèle politique de la Neuvième Symphonie de Beethoven : Alle Menschen werden Brüder. Sa solitude, fruit de l'amour impossible, est l'autre prix à payer, outre le pacte avec le Diable, pour sauver l'art de la conception aristocratique de Zeitblom, celle aussi de Thomas Mann. Alors seulement, il découvre l'organisation non aliénée de la société, en confiance avec l'humanité. Mais il est un malade. «Maintenir assez loin l'un de l'autre les mots vie et Leverkühn $n^{4}$ ", notait Thomas Mann : l'esthétisme de la santé serait «avant-coureur de la barbarie ». Enténébré, son esprit le devient en 1930, avant l'arrivée au pouvoir des nazis, mais entretient un lien étroit avec l'apothéose de la barbarie et dédouane tout autre de la folie. Les valeurs de l'humanisme naissent-elles de cette connivence avec la décadence? Au sein d'une société distraite et massifiée, la maladie, indissociable du génie, s'immisce dans l'œuvre de Manzoni sous la triple forme de la syphilis dans l'acte I (scènes 2, puis 3 et 5 avec le docteur Erasmi), de la méningite d'Écho dans l'acte II (scènes 2, avec le Pédiatre, et 4) et du délire de Leverkühn dans l'acte III, scellant la fin de la modernité. Quant à Zeitblom, il est dans le livret une présence austère, comme une hantise de l'histoire. Il ne lui est guère donné de parler, sinon dans l'épilogue, où Manzoni lui confie de courts fragments récités de la dernière visite à son ami, dont la dernière phrase de l'opéra, ultime datation : « Le 25 août 1940 me parvint [...] la nouvelle que s'était éteinte la suprême étincelle d'une vie qui avait donné à la mienne sa substance essentielle, dans la tendresse, la tension, l'effroi et la fierté5. » 
Manzoni recherche le noyau universel du roman. Onze tableaux, entre ironie, sarcasme et délire... Six, brefs, à l'exception du dernier, dans le premier acte : Leverkühn et les nymphes (1), la syphilis contractée (2), la première visite au docteur Erasmi (3), la composition de l'Ode to a Nightingale (4), la seconde visite au docteur Erasmi (5), le dialogue avec Lui (6), culmination de l'acte. Quatre, dans le deuxième acte : les chants d'Ariel (1), le mal de tête d'Écho et le diagnostic du Pédiatre (2), le monologue bouffon de l'imprésario Saül Fitelberg (3), la révocation de la Neuvième Symphonie de Beethoven (4). Un interlude (Chant de douleur du docteur Faustus), une scène, dans le troisième acte - les hallucinations de Leverkühn, ponctuées par différents personnages (Esmeralda, Écho, Lui et Frau Schweigestill), un groupe et un chœur d'enfants -, et enfin l'épilogue. Autrement dit, à trois reprises au moins (Ode to a Nightingale, chants d'Ariel et Chant de douleur du docteur Faustus), Manzoni compose les œuvres fictives d'Adrian Leverkühn d'après leurs descriptions dans le roman de Thomas Mann.

Dans la scène 4 de l'acte I, Manzoni met en musique, en anglais, quatre fragments de l' Ode to a Nightingale de John Keats, que Thomas Mann évoque au chapitre XXVII, et qu'il associe à la nostalgie, sinon à la mélancolie de la vie méridionale et à la suavité réconfortante d'un monde de lumière. Dans cette composition de Leverkühn, et dans sa réalisation chez Manzoni, pour voix et quatuor à cordes - Manzoni y ajoute percussions et ondes Martenot -, «il s'agissait d'une forme de variations extrêmement artistique, où aucune note, pas plus celle de la voix que des quatre instruments, n'était sans signification thématique ${ }^{6} »$. Cet ultrathématisme, dans sa réfutation du rapport traditionnel entre mélodie et accompagnement instrumental, se souvient du Quatuor op. 10 de Schoenberg et de l'introduction du chant dans ses deux derniers mouvements. De même, dans la scène 1 de l'acte II, où s'exprime l'attachement de Leverkühn pour son jeune neveu, Écho, Manzoni réalise l'hommage à l'enfant, en mettant en musique les chants d'Ariel dans La Tempête (I, 2), Come unto these yellow sands, «bruissant des voix fantomatiques et dispersées de la nature ${ }^{7}$ » et Full fathom five thy father lies, selon l'effectif du roman: soprano, célesta, violon en sourdine, hautbois, trompette avec sourdine et harpe. Manzoni, par la sécheresse de la harpe, des pizzicatos du violoncelle et des notes piquées des vents, atteint ce « réseau murmurant, fin comme une toile arachnéenne, la légèreté aérienne, puérilement suave et troublante d'Ariel ${ }^{8} »-$ une musique de l'éther.

Entre archaïsme et modernité, le Chant de douleur du docteur Faustus, second oratorio d'Adrian Leverkühn, effleure Parsifal, mais surtout Monteverdi, l'esprit du madrigal, la lamentation d'Ariane, liant intimement, à travers la plainte, « incessante, inépuisablement soutenue, avec la plus douloureuse attitude d'un Ecce Homo ${ }^{9}$ ", Faust et Orphée. Une telle lamentation, montéverdienne et leverkühnienne, fonde la musique moderne, expression harmonique par opposition à l'objectivité et aux constructions de la polyphonie, ou plutôt reconstruit l'expression par le retournement de la construction, abstraite, en expressivité, au-delà de toute subjectivité. Décrivant cette œuvre achevée dans l'euphorie, au moment où l'Allemagne s'apprête à rouler « dans la fosse à purin mythique, la boue mythique originelle » du nazisme, Thomas Mann écrit à l'avant-dernier chapitre du roman: "L'écho, la restitution de la parole humaine comme d'un son de la nature, sa révélation en tant que son de la nature, est essentiellement une plainte, le douloureux "hélas !" de la nature devant l'homme et son effort de communiquer son isolement, comme inversement, la plainte des nymphes s'apparente à l'écho ${ }^{10} »$. Lasciatemi morire. Ce chant de douleur s'apparente, en la 
réfutant, à la Neuvième Symphonie. Rétractation de l'hymne à la joie, l'art de Leverkühn culmine, ici encore, dans le renversement, la pensée négative. Car, en un mouvement dialectique, la plus stricte rigueur est garante de la plus libre expression : «Ce style, cette technique, avait-il dit, n'admettraient plus une note, plus une seule, qui dans l'ensemble de la texture sonore ne remplît sa fonction thématique. Il n'y aurait plus une note libre ${ }^{11}$. » L'ordonnancement du matériau, utilisant tous les intervalles, se fait total. Dans la réalisation de Manzoni, alternent une introduction orchestrale, un chœur à six voix, qui, comme l'écrit Thomas Mann, fait fonction de solo et crée l'« identité du multiforme ", un interlude orchestral, action plus violente, traduisant la descente aux enfers, et un chœur à huit voix, s'achevant sur une interrogation, Ach, ach, warum ?, accompagnée d'un violent cri en tutti. En outre, Manzoni embrasse deux traits techniques du roman : l'écho, dans l'écriture contrapuntique et surtout dans la forme, où toute variation, statique, réverbère la précédente ; et l'utilisation du retard comme procédé rythmique dans le second chœur, sans dynamisme, ni développement, mais en élargissements progressifs et en cercles excentriques.

La construction du Docteur Faustus manzonien repose sur le nom de Hetaera Esmeralda, facteur d'unification intervallique rapporté par Thomas Mann dans le chapitre XIX : h, e, a, e, es $+d$, oublié par Mann $(=s i, m i, l a, m i, m i$ bémol + ré, selon la notation musicale allemande). Une citation de La Tempête de Purcell (dans la musichetta de l'acte II, introduction aux chants d'Ariel), une chanson enfantine imaginaire (à la fin de cette même scène), la tête de l'Arietta de l'op. 111 de Beethoven (en souvenir de Kretzschmar) sont d'autres éléments sporadiques, matériaux de construction témoignant de ruptures stylistiques, tout comme la fameuse dissonance de la Neuvième Symphonie, les vingt-et-une impulsions extraites de cette symphonie, l'utilisation structurelle de la métrique du texte, telle que la pratique dans le roman le Chant de douleur du «Docteur Faustus », et son principe dodécaphonique sur l'alexandrin suivant: "Je meurs à la fois en bon et mauvais chrétien ${ }^{12}$. » Ces éléments, tirés du roman, se creusent encore. Dans son Journal du Docteur Faustus, relevons cette assertion de Thomas Mann: "Rien de noble n'aurait surgi après Purcell ${ }^{13}$. » Et sur l'Arietta : «La conversation qui suivit roula sur l'humanité en tant qu'élément chtonique épuré, les liens entre Beethoven et Goethe, l'humain en opposition romantique avec la société et la convention (Rousseau) et comme révolte (la scène en prose dans le Faust de Goethe). Après quoi Adorno me joua d'un bout à l'autre, tandis que je me tenais auprès du piano à le regarder, la sonate op. 111, de façon extrêmement instructive. Jamais je n'avais été aussi attentif ${ }^{14}$. » Dans le chapitre VIII, Thomas Mann insinua, comme une secrète marque de gratitude, le nom de Wiesengrund, en hommage à Theodor W. Adorno, l'aide, le conseiller, l'instructeur plein de sympathie, selon les termes du romancier, admirateur du radicalisme et du "sans-culottisme musical ${ }^{15}$ » du philosophe. Thomas Mann avait toutefois une idée poétique précise du matériau qu'Adorno lui détaillait techniquement dans des descriptions parfois reprises littéralement, en raison de leur intériorisation des intentions de l'écrivain. Mais ce que les allusions et les citations musicales de Manzoni prolongent, en sus de la diversité des langages et des stylèmes, c'est une tension dialectique, au sein d'une forme fermée, close, entre deux modèles opposés, celui de l'intégration organique, sur laquelle se fonde avant tout le roman, et celui du montage de fragments, où la substance cède le pas à la liaison.

Docteur Faustus est une réponse lointaine à une œuvre antérieure de Manzoni, Per Massimiliano Robespierre. Théâtre d'idées, sinon d'idéologie, cette action 
scénique reposait sur un dense réseau de fragments littéraires, parfois contradictoires : discours, lettres, poèmes et articles de Robespierre et de ses contemporains, mais aussi jugements ultérieurs, textes politiques, philosophiques et poétiques, sur la Révolution française et sur celui qui en fut l'un des principaux artisans. Sans personnage réel, hormis la sœur de Robespierre, ce montage étranger aux conventions de l'opéra renonçait à la stricte narration linéaire. La ligne qui mena Manzoni de Per Massimiliano Robespierre à Docteur Faustus suivit des voies sinueuses aux fondements historiques. Entre 1794, date de la chute de Robespierre et de l'échec d'une tentative d'assurer à la Révolution française un aboutissement plébéien et démocratique, et 1799, année de la fin du Directoire et de l'avènement de la dictature militaire du Premier consul, naît le romantisme allemand, en lutte contre la modernité, la démocratie, le droit, l'égalité civile, la liberté individuelle... Hostile à l'Aufklärung, l'alémanité est au cœur du romantisme allemand et de son visage crépusculaire dans le roman de Thomas Mann, lequel affirma la nécessité d'une nouvelle conscience nationale. «Classicisme et romantisme s'occupent en Allemagne des mêmes problèmes dont l'aspect antérieur a été profondément modifié par la victoire de la Révolution française; mais pour les principaux de ces problèmes, le romantisme propose une solution différente et diamétralement opposée à celle des classiques ${ }^{16}$.» Lukács souligna qu'un tel romantisme ne visait nullement au rétablissement d'un ordre précapitaliste, mais à sa transformation, sans détruire les privilèges féodaux auxquels se rattachait l'Empire de 1871. Le mélange de "robuste modernisme", d'« efficacité avancée » et de "rêve du passé ", cet esprit à la fois armé devant la réalité et dressé contre elle par l'ivresse idéologique et les légitimations du mythe, Thomas Mann le nomme romantisme technicisé ${ }^{17}$. Et si le romantisme a poétisé la morale politique allemande, cette alémanité comme germanité doctrinale, et son essence esthétique, et à tout le moins solipsiste, s'opposent d'une façon intransigeante au social.

Se donnant pour une aristocratie de l'esprit, qui serait de plus liée aux idées traditionnelles de peuple et de communauté, la culture allemande prétendit trouver un substitut local à la Révolution française dont elle travestissait et bafouait les idéaux. Après l'avoir longtemps dissociée de la politique, Thomas Mann analysa, au sein de l'alémanité, l'idée de culture, laquelle se considère investie d'un destin d'élection dans l'accomplissement des fins supérieures de l'humanité. Dans "Culture et socialisme », il dévoila la faiblesse idéologique et morale des Allemands devant les hypnoses autoritaires et tyranniques. Dans son acception germanique, la culture serait une exaltation d'une individualité intérieure prussifiée, d'une soumission à un ordre autoritaire introjeté qui renvoie à des valeurs de "caractère religieux, c'est-à-dire essentiellement asocial, égoïste, individuel ", et d'une "Kultur " appelée à devenir l'«idée-force de l'idéologie de guerre allemande ${ }^{18}$ ». Ses refuges, l'intellectuel, passif, les trouve nécessairement dans la beauté morale et l'illusionnisme spéculatif. Leverkühn personnifie la critique la plus radicale de cet alémanisme irrationnel, responsable de ces fautes dont il porte le poids. Aussi Thomas Mann écrivit-il, implacable : « Le national-socialisme, dans toute son abjection empirique, ineffable, est la conséquence tragique de cette innocence mythique en politique qui caractérise l'esprit allemand ${ }^{19}$. » À travers ce règlement de compte féroce de l'alémanité, dont $L e$ Docteur Faustus constitue l'acmé, se précise la dimension nietzschéenne du roman - Nietzsche, autre modèle tragiquement biographique, dont Manzoni redécouvrit l'œuvre au moment de la composition de son opéra et sur des fragments duquel il écrivit ensuite Il deserto cresce, tre metafore da Friedrich Nietzsche (1992), 
pour chœur et orchestre. Or, la musique, art de l'ambivalence érigée en système, exprimerait l'essence de l'alémanité et son rapport fondamental avec le monde, sa révolte contre la civilisation et son inaptitude au politique. La révocation de la Neuvième Symphonie prend alors un autre sens. Car Beethoven est celui qui " commence la musique d'expression » et qui « incarne le péché de la musique tombant du plan céleste au plan humain ». À ce sujet, Thomas Mann bénéficia de remarques d'Adorno et de Hanns Eisler sur le langage musical et la polyphonie des dernières partitions de Beethoven, remarques reprises dans les premiers chapitres du roman et exposées par Wendell Kretzschmar.

Malgré d'évidentes affinités avec la technique du leitmotiv dans la construction du roman, l'ironie de Thomas Mann à l'encontre de Wagner, vilipendant « le caractère illusionniste de l'œuvre d'art visant à la réalité », fait écho à la fantasmagorie wagnérienne d'Adorno tentant de démontrer son affinité avec l'idéologie nazie. Dans une lettre datée du 30 octobre 1952, Thomas Mann citera en retour l'Essai sur Wagner où le philosophe se demande «si l'exigence nietzschéenne de la santé vaut mieux que la conscience critique à laquelle s'élève la faiblesse grandiose de Wagner dans la communion avec les forces inconscientes de sa propre déchéance ${ }^{20} »$. Musicien de la catharsis et de l'emphase, Wagner invoque un passé obscur et participe d'une germanisation idéologique, où la tradition historico-culturelle du passé allemand, voire ce que l'on pouvait tenter d'y annexer, devient son essence, l'alémanité. Sa « révolution archaïque " mêle indistinctement éléments réactionnaires et révolutionnaires, tout en ignorant le social, et ses mythes mythologisent la mémoire nationale, se constituent dans la dimension et dans la fonction du muthos, et rassemblent forces et orientations d'une identité souterraine. Brutalité, naïveté, dialectique de l'instinct et de la domination: selon Adorno, une telle limitation de la raison discrédite l'intellection, favorise un irrationalisme anhistorique qui rend suspect toute idée de progrès. Dans " Destin et Devoir ", Thomas Mann s'interroge sur le travestissement, la défiguration de Wagner par les nazis, et juxtapose l'épuisement de la musique allemande postwagnérienne et l'autodestruction de l'Allemagne pendant la même période, son arrogance blasphématoire stylisant la culpabilité en tragédie. Le destin de Leverkühn et celui d'Adolf Hitler sont indissociables: on ne sort de l'entre-deux en réveillant l'antique puissance du mythe qu'au prix de la damnation. À la critique de l'alémanité, à cette influence de Nietzsche quant à la dichotomie de la culture allemande, répondent dans l'opéra de Manzoni le choix des langues et l'utilisation référentielle de la traduction italienne, esquivant ainsi la "densité massive» du roman de Thomas $\mathrm{Mann}^{21}$. Car Faust est devenu pour ainsi dire un personnage constitutif de la culture européenne. En lui s'exprimerait l'exigence non d'un seul être, ni d'un seul peuple, ni d'une époque déterminée, mais une aspiration profondément universelle, la "dialectique de l'humain et des forces de la Terre (barbares)"22 ». Lui, le Diable, ne disait-il pas, et ne chantait-il pas dans la scène 6 de l'acte $\mathrm{I}:$ « Je suis allemand, il est vrai, allemand jusqu'à l'aubier, mais tout de même de la vieille souche, supérieure, à savoir cosmopolite de cœur ${ }^{23} »$ ?

Un dernier mot sur le dialogue avec le Diable et la condamnation que Leverkühn, à la différence du Faust traditionnel, accepte obtorto collo : le tort est déjà fait, et la contagion contraint le musicien à prendre acte des conditions du pacte. Cette scène, la sixième et dernière de l'acte premier, cristallise la dimension politique de l'opéra, où se dessine la construction intellectuelle, analytique, sinon scientifique de l'altérité et du génie démoniaque. Selon Manzoni, Satan, le Diable, la trine figure du Lui, sauve Thomas 
Mann du dilemme entre rester soi-même et reconnaître une nécessité révolutionnaire, en contradiction avec une sensibilité bourgeoise très enracinée dans sa nature, sans donner un visage au moderne. L'individu assume des tâches immenses, excédant la contingence de son moi. Dans l'œuvre de Manzoni, le sujet est vecteur d'une philosophie de l'histoire d'essence marxiste, dictée par les lois et les tendances du matériau musical. La violence des rapports sociaux, dont le matériau est porteur, conditionne objectivement, mais obscurément le jugement du sujet, le processus de formation de l'œuvre connaissant l'influence du devenir social - dans ce contexte, Lukács avait eu l'intuition de l'artiste réactionnaire dans la vie et " réaliste », et donc progressiste, dans son œuvre. Le Diable, «symbole de la sécularisation de la musique, du passage de celle-ci au rationnel, à la conscience critique de soi ", perd de la sorte ses connotations séculaires. Seule reste la voix glaciale de l'homme, ce "froid brûlant" diabolique évoqué par le mystique allemand Franz von Baader. "Le pacte avec le diable, c'est cela : le grand art, affirme le diable, ne peut être produit dans cette société décadente que par la solitude, l'isolement total et la totale absence de cœur ", écrivait Eisler, qui précisait, dans un article sur Johann Faustus rédigé à la mort de Schoenberg, que «la méthode de construction du système dodécaphonique prodigue une froideur ardente ou une chaleur glacée, de la même manière que le feu brûle comme les grands froids ${ }^{24} »$. Inscrit dans le roman, ce dodécaphonisme, symbole de la désorientation du monde, art du désespoir, renouvelle et libère les contenus musicaux, mais à travers la contrainte. La musique sort avec Le Docteur Faustus de l'ineffable, des métaphores brumeuses, de la « caverne de l'inconscient " (Manzoni), et préfigure la constitution d'une nouvelle société. Sa lucidité critique et sa rationalité constructive sont en quelque sorte déplacées dans la préformation d'un matériau musical auquel souscrit Manzoni. «En un certain sens, plus grossier et sous le rapport de la matérialité des sons, le travail est déjà accompli avant même que ne commence la composition et celle-ci peut se donner libre cours, c'est-à-dire céder à l'expression ainsi récupérée audelà du plan constructif ou à l'intérieur de sa rigueur la plus absolue ${ }^{25}$ ». L'intégration de toutes les dimensions sonores, leur indifférence réciproque en vertu de leur parfaite organisation, trouve un écho dans la notion de paramètres bien avant les années cinquante et les expérimentations du sérialisme dit de Darmstadt. Mais si le dodécaphonisme, issu de Brahms et de Wagner, inscrit selon l'auteur dans une généalogie remontant aux Franco-flamands, est un phénomène éminemment allemand, son devenir historique, ici italianisant, évide-t-il la substance historique de ses fondements?

\section{NOTES}

1. En 1955, Giacomo Manzoni avait écrit dans la revue Diapason l'article « Pubblicazione del Doktor Faustus e la polemica tra Arnold Schoenberg e Thomas Mann », et en 1986, dans les Quaderni di Musica/Realtà, un article sur Ferruccio Busoni et Thomas Mann « Il duplice volto di Faustus ».

2. Mann (Thomas), Lettre à Theodor W. Adorno du 30 décembre 1945, op. cit., p. 211.

3. Lukács (Georg), Brève histoire de la littérature allemande, Paris, Nagel, 1949, p. 236. 
4. Mann (Thomas), Journal 1940-1955 (6 septembre 1944), Paris, Gallimard, 2000, p. 201.

5. Mann (Thomas), Le Docteur Faustus, op. cit, Épilogue, p. 666.

6. Ibid., chap. XXVII, p. 355.

7. Ibid., chap. XLIV, p. 615.

8. Id.

9. Ibid., chap. XLVI, p. 635.

10. Id.

11. Ibid., chap. XLVI, p. 636.

12. Ibid., chap. XLVI, p. 639.

13. Mann (Thomas), Le Journal du « Docteur Faustus », op. cit., p. 72.

14. Ibid., p. 45.

15. Ibid., p. 42.

16. Lukács (Georg), Brève histoire de la littérature allemande, op. cit., p. 76-77.

17. Mann (Thomas), «L'Allemagne et les Allemands », op. cit., p. 358.

18. Mann (Thomas), «Culture et socialisme » (1928), in L'Artiste et la Société, Paris, Grasset, 1979, p. 205.

19. Mann (Thomas), «Défense de Wagner » (1940), in Wagner et notre temps, Paris, Pluriel, 1978, p. 179.

20. Adorno (Theodor W.), Essai sur Wagner, Paris, Gallimard, 1966, p. 209. Cf. Mann (Thomas), Lettre à Theodor W. Adorno du 30 octobre 1952, in Lettres 1948-1955, Paris, Gallimard, 1973, p. 299. 21. Cf. Manzoni (Giacomo), «Il lungo cammino del Doktor Faustus» (1987), in Scritti, Florence, La Nuova Italia, p. 118-119.

22. Mann (Thomas), Journal 1940-1955 (4 octobre 1943), op. cit., p. 167.

23. Mann (Thomas), Le Docteur Faustus, op. cit., chap. XXV, p. 308.

24. Eisler (Hanns), « Notizen zu Doktor Faustus», in Wer war Hanns Eisler, Berlin, deb, 1983, p. 172-176.

25. Mann (Thomas), Le Docteur Faustus, op. cit., chap. XXXVII, p. 521.

\section{AUTEUR}

\section{LAURENT FENEYROU}

CNRS-EHESS 